\title{
20
}

\section{"SHESHET"* - Decision support system for guidance and placement of enrolling high school students}

\author{
Poria Kalay and David Chen \\ School of Education, Tel-Aviv University, Ramat Aviv, P.O.B, 39040, 69978, Tel Aviv, Israel
}

\begin{abstract}
Many high schools have to tackle the problem of placement of the students who want to enroll in them, and into tracks, classes and vocational programs. The decision-making process of matching between individual aspirations and the system capabilities is known to be complicated and ineffective. This paper describes a decision support system developed to assist the principal and the counselors in the placement process and the integration of the system in school.
\end{abstract}

Keyword Codes: H. 1.2, D.1.1, C.5.2

Keywords: User/Machine System, Applicative Programming, Minicomputers

\section{INTRODUCTION}

When students graduate from junior high school choices must be made with regard to high schools they want to enroll in, the kind of program (or track) they can select and the specific class they will be placed in. At the same time, the school principal, the counselors and the professional teachers have plenty of decision-making to do with regard to registration selection and placement of the new students.

The process of matching between the individual aspirations and the system capabilities is known to be complicated, intuitive, time-consuming and ineffective. The case of vocational programs is even more complicated as the decisions made by either side will, to a large extent, affect the future career of the individual student after school.

The problem at hand can be defined as an optimization problem: Is it possible to improve contemporary practices of educational decision-making in the guidance stage when junior high students opt for technology school, compromising between individual characteristics and school capability. This problem can be defined within the frameworks of the theories of adaptive education $[1,2]$ or effective schooling [3].

Since the decision-making process is so central to the abovementioned problem, we examined the possibility that utilization of an appropriate information technology to support the guidance process will improve it significantly.

Our approach was to develop and utilize a decision support system (DSS) based on individual and school data bases, a set of educational decision-making rules (elimination by aspects) to provide the school staff with a tool that would improve the placement process into vocational programs with clear and standardized criteria that can be justified. The DSS would improve the ability to comply with the individual needs, and increase administrative capacity. The advantage of our approach is that while the computer undertakes much of the information

* "SHESHET" is the Hebrew for a decision support system named after Robinson Crusoe's servant Friday. 
complex educational decision-making, it leaves both the criteria and the final decision-making in the hands of the school staff. We will describe the DSS developed and initial evaluation of the integration of the system in school.

\section{LITERATURE REVIEW}

Computer Assisted Guidance (CAG) systems have been in existence since the early sixties. These systems can be broadly divided into three groups:

(1) Information retrieval systems - in which the task of the computer is basically to find the "page" on which the information required by the client appears.

(2) Information processing systems - in which the task of the computer is to identify educational or occupational opportunities which satisfy a number of criteria proposed by the client.

(3) Learning systems - which concern retrieval and processing of information in a way that helps clients to learn relevant skills and concepts [4].

Most CAG systems can be characterized as follows:

(1) The system includes data bases.

(2) The system includes interface for the user.

(3) The guidance is projected from the point of view of the individual and not from that of the educational system.

Existing systems do not include a broad decision-making component [5].

In the early sixties new possibilities were presented to computer users: new technologies offered quick access to data and information, and made it possible for the user to have a dialogue with the computer. This opened new horizons to managers of organizations and turned the computer into a tool capable of assisting in quick decision-making.

Simon [6] divided decisions into two types:

(1) Structured decisions, which are programmable.

(2) Unstructured decisions, which are non-programmable.

A decision is purely structured if the whole process of decision-making can be described in detail before the decision is made. The fact that a computer can make structured decisions just like human beings turns it into an efficient tool in making decisions of this type. Therefore, computer utilization in making structural decisions is widespread, the burden of making such decisions lifting off the manager or the person who was previously in charge.

Note that defining a decision as structured or unstructured is describing two extremes. In reality there is a wide spectrum of decisions ranging from purely structured to purely unstructured. Decisions which are partially structured and partially unstructured are called semistructured decisions.

According to Gorry and Scott Morton [7], most of the important decisions made in an organization are semi-structured. These authors introduced the term Decision Support System (DSS) to describe systems made to support semi-structured decisions. However, while the computer's main contribution in structured decision-making is to increase the efficiency of the process, DSS help increase the effectiveness of decision-making itself. The main purpose of DSS is not, therefore, to save working hours, but to help in making a better decision.

The DSS supports tasks in which some of the functions are better handled by the system and others are better handled by the manager. The "SHESHET" system for guidance and classification of pupils is based on two models:

(1) Simon's model of "Limited Rationalism" [8]

This model assumes that the number of alternatives examined in the decision-making process is limited, and the one alternative most satisfying is chosen [6]. This theory is of great importance regarding the development of DSS, because of the unique nature of these systems which are based upon man-machine interrelations. 
By definition, the DSS must lead to full cooperation between man and the computer during the decision-making process. The formal division of decision-making processes into steps, which can be defined to the computer so it can plan its assistance in each one of them, has gained great prominence. The idea is that if we track a person's decision-making process, we would probably find a definite process by which he achieves decision-making. Once this process is identified we could install into the computer those functions which would assist a person in each step of the process. If the user wishes to skip some steps while making a certain decision, he can use only those functions involved in steps that are relevant.

(2) Tversky's model of Elimination By Aspects EBA [9]

According to this model alternatives consist of certain aspects differing in their relative importance level. The decision-maker determines certain level in each criterion below which the alternative is ruled out. The decision-maker examines all the alternatives according to the most important criterion and rejects all those that did not make the minimum point. In the next stage he will examine only the remaining alternatives, this time according to the second most important criterion, and rule out, again, those that don't stand up to it. This goes on until the end of the process [9].

In this method of decision-making, there is no compensation for "weak criteria" by "strong criteria" of a certain alternative. This means that a certain alternative which is below a minimum according to one criterion, will be ruled out even if it perfectly suits some other criteria. The "SHESHET" system overcomes this difficulty by considering exceptional criteria. The advantage of the EBA model is its relative simplicity, the fact that there is no need for calculations and the way it suits common sense.

\section{THE "SHESHET" SYSTEM}

\subsection{Description of the system}

The system comprises the five following components:

(1) The data base.

(2) The computerized decision support system.

(3) The student placement plan.

(4) The school guidance operation.

(5) The operational student placement.

We shall describe the five components in detail and the way the DSS technology integrates in the school to provide guidance for the high school freshman.

The data necessary for decision-making are available in two files, the school file and the student file. The school file is small and simple: it consists of the names of the programs the school offers (mechanics, electricity, secretarial, automechanics, etc.), the number of classes per program, qualitative dimensions of the classes, and the list of school resources - such as workshops, science labs, computer stations, teachers, budget, etc.

The student file is large and complicated. It is large because it contains the data of the entire student body, and it is complicated because the student data are not standardized and updated.

Both ideological and sociological controversies interfere with psychometric data collection, achievement measurements, personality traits and preferences (which are critical for the guidance process), thus causing the information base necessary for the educational decision-making process to be both insufficient and invalid.

In this study, we have accepted the individual data available in the school "as is", while keeping in mind the resulting limitations.

The decision support system was developed utilizing "Hypercard" and a Macintosh SE/30 computer. The engine driving the system was based on the "Elimination By Aspects" principle derived from Simon [6] and Tversky's [9] decision-making theory.

Our basic analysis of the decision-making process led us to believe that it followed the "semi structured" model. The relevant individual data (1a) (See Figure 1) are sent as an update into the 
DSS (2). The elimination criteria predetermined by the educational staff (such as upper and lower limits of school grades, psychometric profiles, preferences, etc.) are used as rules that support the placement of each individual in a specific class and program.

At the end of the program's decision-making, the system generates an output (3) that consists of several alternatives, $P_{1}, P_{2}, \ldots P_{n}$, for consideration of the staff (4). The decision-making now is entirely in the hands of educational team which examines the nature of the different programs, the kind of students that have entered each learning group and the degree of strain that each alternative has put on the school. Individual aspects are also considered: individuals who request changes can apply and justify, and changes of individual schedules can be examined through several alternatives.

At the end, the guidance process is completed by a final decision or alternative Px (5). Upon decision, the individual students and their parents are informed about the decisions concerning their studies while the criteria for the decision are clearly defined and can be justified.

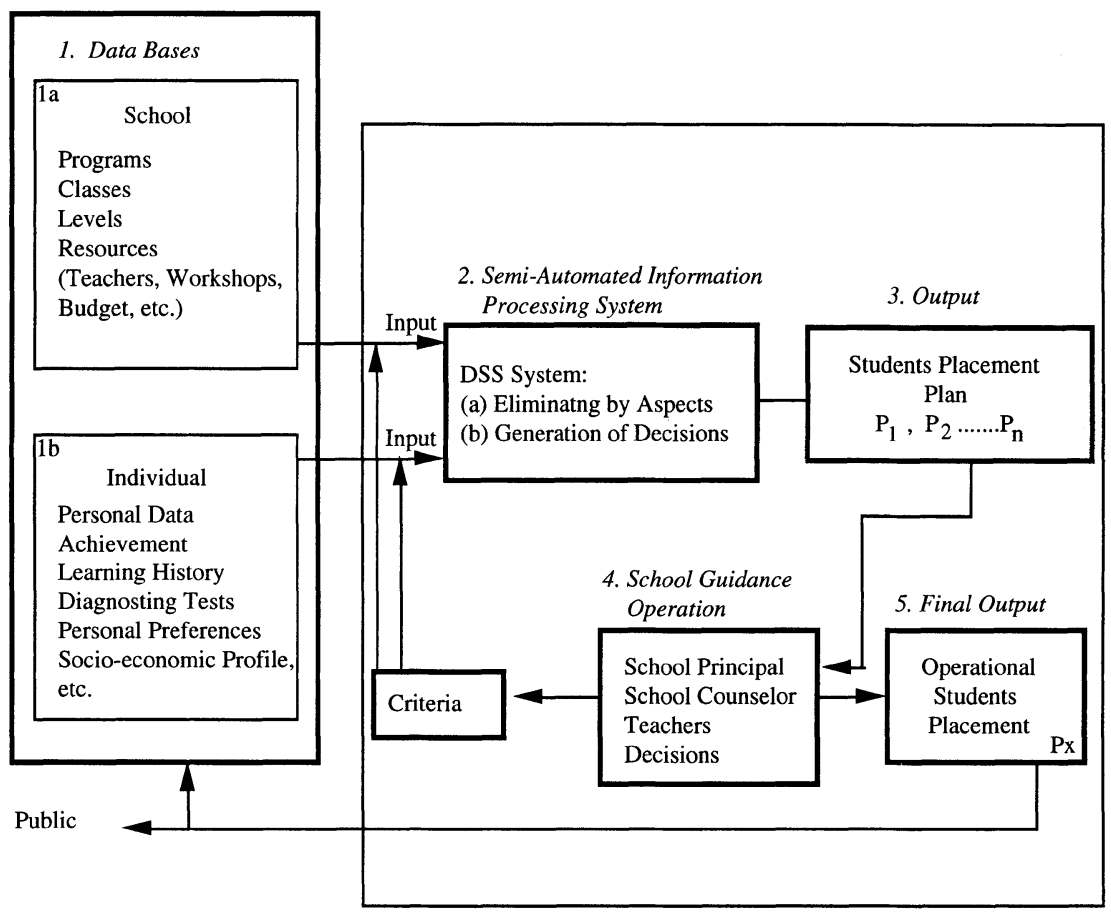

Figure 1. The "SHESHET" Decision Support System

\subsection{Description of the system}

The prototype of the system was developed and tried out in a technological school in Israel during the academic year 1989/90. The school is of medium size - 28 classes, including 700 students.

\section{System adjustment stage}

The prototype of the decision support system for guidance and placement of students was adjusted to the demands of decision-making personnel in the school (principal, counselors). 
The data base of the system was determined in cooperation with the school counselors with reference to:

1) Structure of the student's card.

2) The files within the student's card.

3) The nature of the different files (number, words, group, record).

\section{Data collection stage}

In this stage all the data concerning the students was collected and keyed into the computer.

The data included:

1) Personal details.

2) Previous achievements record.

3) Entrance (classification) exams arranged by the school.

4) Students' preference for different vocational programs.

\section{Decision-making stage}

The process of decision-making can be divided into three:

1) Guidance according to students' capabilities.

2) Guidance according to opportunities offered by the school.

3) Guidance according to limitations of the school.

Each one of these stages is supported by a relevant part of the software.

\section{Dialogue stage}

The decision-making team chose the first criterion according to which it wanted to start classifying by elimination for the highest standard in the school.

On the basis of the distribution, the team decided the range of the criterion.

The system reviewed data on all students, and a numbered list of those who fulfilled the criteria was produced.

At this stage the decision-makers could choose between four possibilities:

1) Changing the range of the first criterion.

2) Canceling the first criterion and classifying students according to a different one.

3) Checking the distribution of students according to the first criterion against any other criterion.

4) Accepting the first criterion and its range which had hitherto led to a temporary status, and turning them into permanent ones.

Continuation of the placement process depended on the decision made in the first stage.

The decision makers chose another preferable criterion and repeated the whole process as mentioned above. After some alternatives were examined, the decision-makers decided that the group formed according to the criteria they presented suited the course they wanted to determine.

They determined the course (level) and placed the students in vocational programs according to their preferences.

At the end of the classification to the highest standard, an examination was arranged by classifying according to different criteria in order to reach students who could not fulfill the first or second criteria but fulfilled the others.

In the next stage a classification to a lower standard was carried out in which the above mentioned process was repeated.

The student population was similarly placed in courses of study and programs.

\subsection{Initial evaluation of the system}

The prototype of the system has improved and the system worked in an organized manner in the years 1991-92 and 1992/93. The fndings of the research concerning the effect of the system will be published in a paper to follow. The initial evaluation indicated an efficient and effective integration of the system in the school. The system enables handling and processing of a great amount of data, and the placement and classification process now takes hours whereas without the system it took weeks. 
The system enables the altering of criteria and the comparison between alternatives, and brings about standardization and objective decisions justifiable.

The system facilitates new options in the midst of the decision-making process: receiving divisions of the classes we built; identification of special groups of students for special treatment and learning; division of the students into groups according to their learning ability in certain subjects, for example, English or mathematics.

The initial findings indicate that the drop-out number of students has been reduced, the transference from one learning level to another has become lower and that there has been an improvement in the achievement of the students.

\section{REFERENCES}

[1] R. Glaser, Adaptive Education: Individual Diversity and Learning, Holt, Rinehart and Winston Psychology Series, New York, 1977.

[2] R.E. Snow and L. Corno, Handbook of Research Teaching, 3rd edition, New York, Merlin \& Wittrock, (1986) 605.

[3] D. Reynolds, B.M.P. Greemers and T. Peters, School Effectiveness and Improvement, Groningen, The Netherlands: Rion Insttiute for Education Research, 1989.

[4] A.G. Watts, Int. J. Adv. of Counseling, 9 (1986) 145.

[5] M.R. Katz and L. Shatkin, The Couns. Psychol., 11(4) (1983), 15.

[6] H.A. Simon, The New Science of Management Decision, Prentice-Hall, New Jersey, 1960.

[7] G.A. Gorry and M.S. Scott Morton, Sloan MGT Rev., Fall, (1971).

[8] H.A. Simon, (ed.), Models of Man, Wiley \& Sons, New York, (1957) 241.

[9] A. Tversky, Psychol. Rev., 79, (1972) 281. 